\title{
Análisis de la estructura económica regional del espacio bifronterizo Tacna-Arica (2007-2017)
}

\author{
Analysis of the regional economic structure of the Tacna-Arica bi-border \\ area (2007-2017)
}

\author{
Edwin Ismael Palza Chambe \\ Universidad Nacional Jorge Basadre Grohmann \\ Tacna, Perú \\ epalzach@unjbg.edu.pe \\ https://orcid.org/0000-0001-6411-2907
}

[Presentado: 01/02/2021, Aceptado: 08/03/2020]

\section{RESUMEN}

Desde que en 1929 se suscribió el tratado de límites entre Perú y Chile, determinándose que Tacna regrese a posesión de Perú y Arica continuara en administración del gobierno chileno, se generó el rompimiento de un espacio común y que de manera sinérgica se perfilaba como uno de los más importantes del Pacífico. Esto generó el deterioro de la economía de ambas regiones, las cuales además fueron agudizadas por las condiciones de contexto y la exposición a grandes crisis presentes a lo largo de la mayor parte del siglo XX. La metodología de la técnica de análisis regional permitió determinar la retracción de la dinámica de la región Tacna y el crecimiento de la observada en Arica y Parinacota en el período de análisis 2007-2017, en una expansión sustentada fundamentalmente en el crecimiento coyuntural de sectores como la minería y la labor gubernamental, las cuales han incorporado a una importante proporción de la población económicamente activa de esta región.

Palabras clave: Economía Tacna-Arica, empleo, frontera, producción.

\begin{abstract}
Since 1929, when the border treaty between Peru and Chile was signed, determining that Tacna would return to Peruvian possession and Arica would continue to be administered by the Chilean government, a common space that was synergistically emerging as one of the most important in the Pacific was broken up. This generated the deterioration of the economy of both regions, which were also aggravated by the contextual conditions and the exposure to major crises present throughout most of the 20th century. The regional analysis technique methodology made it possible to determine the retraction of the dynamics of the Tacna region and the growth of that observed in Arica and Parinacota in the 2007-2017 period of analysis, an expansion sustained fundamentally by the cyclical growth of sectors such as mining and government work, which incorporated an important proportion of the economically active population of this region.
\end{abstract}

Keywords: Economy Tacna-Arica, employment, border, production

(C) Los autores. Este artículo es publicado por la Revista Economía y Negocios de la Universidad Nacional Jorge Basadre Grohmann. Este es un artículo de acceso abierto, distribuido bajo los términos de la Licencia Creative Commons Reconocimiento 4.0 Internacional. (https://creativecommons.org/licenses/by/4.0/deed.es), que permite a terceros utilizar lo publicado siempre que mencionen la autoría del trabajo, y a la primera publicación en esta revista. 


\section{INTRODUCCIÓN}

El espacio geográfico colindante entre Perú y Chile aglutina dos unidades espaciales administrativas colindantes: el departamento de Tacna (Perú) y la región Arica y Parinacota (Chile), que ocupan superficies de 16 $076 \mathrm{~km}^{2}$ (Banco Central de Reserva del Perú, s.f.) y $16873.30 \mathrm{~km}^{2}$ (Biblioteca del Congreso Nacional de Chile, s.f.) respectivamente.

Son espacios geográficos de casi similar tamaño, ocupados por 631510 personas (de las cuales el $60.08 \%$ se encuentran localizadas en el lado peruano y $39.92 \%$ en territorio chileno), lo que define una densidad de 19.16 personas $/ \mathrm{km}^{2}$ (Registro Nacional de Identificación y Estado Civil, 2021), (Biblioteca del Congreso Nacional de Chile, s.f.).

La longitud de la línea fronteriza que los separa es de $169 \mathrm{~km}$ (Instituto Nacional de Estadística e Informática, 1998); y las ciudades capitales de ambas regiones se encuentran unidas por la denominada carretera Panamericana que tiene $58 \mathrm{~km}$ entre ellas. En medio, se encuentra ubicado el único puesto fronterizo entre ambos países denominado Chacalluta-Santa Rosa, por el cual transitan 3303264 peruanos y 3627945 chilenos, definiendo a esta dependencia como la segunda en mayor movimiento en todo el Perú, solo superada por el Aeropuerto Internacional Jorge Chávez(Superintendencia Nacional de Migraciones, 2020).

De los más de 6.5 millones de traslados, y considerando lo planteado por Gárate (2016), más de 2/3 partes de estos corresponden a residentes de Arica que se trasladan hacia la ciudad de Tacna, generalmente por un período menor a 1 día, básicamente orientados a las atenciones de salud, actividades de ocio o comerciales. Existe también un importante flujo de peruanos que se movilizan hacia Arica, fundamentalmente hacia el valle de Azapa donde desarrollan actividades laborales; así, Tapia (2015) citando a la Policía de Investigaciones de Chile define que al año 2000 se registraron por los distintos puntos de control fronterizos de la I Región de Tarapacá (la cual incorporaba en ese momento a la Región Arica-Parinacota) los ingresos de 184515 peruanos y bolivianos; así como las salidas de 224426 personas de esta misma nacionalidad; ya para el año 2010 esta cifra creció más de seis veces su valor. Crecimiento básicamente explicado por la modernización de los valles de Lluta y Arica que resultan atrayentes debido a la alta proporción incorporada de fuerza laboral en períodos de cosecha.

Esta continua dinámica ha determinado que Tapia, Liberona y Contreras (2017) definan a esta región como un "territorio circulatorio", lo que implica que más que una frontera tradicional esta zona funciona en la práctica como un espacio contiguo.

Pero esta dinámica no es nueva, Rosenblitt (2018) plantea que Tacna y Arica constituyen, desde su nacimiento en los albores de la Conquista, un espacio propio y común que se vinculaba con los enclaves mineros y centros urbanos localizados en el Altiplano, en lo que se denominó el circuito plata-azogue. Sin embargo, esta dinámica no determinó la irrupción de grupos empresariales regionales propios, sino hasta 1780 cuando se producen reformas promovidas por la monarquía borbona que abolían los corregimientos, y de los funcionarios coloniales que monopolizaron la distribución de manufacturas provenientes de la metrópoli y orientada hasta el altiplano, dejando este espacio libre a los comerciantes locales (Moreno, 1977).

Este proceso ganó en dinámica con la apertura del puerto de Arica para el intercambio con otros puertos imperiales, decretado en 1778, lo que disminuyó adicionalmente la importancia específica del binomio LimaCallao. Así, Rosenblitt (2013) plantea la alta importancia y las enormes perspectivas que apreciaba el espacio al que denominó "tacnoariqueño".

La materialización de la independencia del Perú, tras años continuos de conflicto, devino en una severa crisis para Tacna y Arica, lo que se suscitó ya en la naciente república. Sin embargo, para la década de los 30s del siglo XIX, la actividad comercial empieza a florecer y el carácter complementario de las dos ciudades se ve remarcado y apuntalado por la declaración de Arica como Puerto Mayor en 1825 (Yábar, 2019); a pesar que el proyecto de la Confederación Perú-Boliviana (desarrollada en esos años), y al que la mayor parte de empresarios de la región se plegaron, fue finalmente disuelta. 
El siglo XIX estuvo marcado por consolidar el espacio de la región surandina con Arica como el puerto de embarque y descarga de productos, y Tacna como lugar de almacenaje, la cual sufre abruptamente el desarrollo del conflicto entre Perú y Chile. Concluyendo con la victoria de este último y, en atención a lo determinado en el Tratado de Ancón de 1883, Arica y Tacna pasan a su posesión por una década, para luego someter a un plebiscito la definición de la soberanía del territorio.

Zanjada la discrepancia por la firma del tratado de Lima de 1929 que determina que Tacna regrese a posesión de Perú, y Arica continuará en administración del gobierno chileno, se determinó el rompimiento de un espacio que había permanecido más de tres siglos como uno.

El rompimiento de esta unidad económica y social devine en el deterioro de la economía ariqueña que vivía casi del tránsito de mercaderías y pasajeros de Bolivia y en menor escala del sur del Perú, y con una escasa actividad productiva casi centrada en la pequeña agricultura de sus valles. Esta situación solo cambió vertiginosamente con la creación de la Junta de Adelanto de Arica y la creación del Puerto Libre, ya en los años 50s del pasado siglo (Pizarro y Ríos, 2005).

En el caso de Tacna, el panorama no fue muy diferente, lo cual fue agudizado por la crisis internacional iniciada el mismo 1929 y que se multiplicó en razón a la contracción comercial proveniente y destinada hacia Bolivia, la cual se hallaba inmersa en la Guerra del Chaco; y posteriormente, al estallido de la segunda guerra mundial (Díaz y Bustos, 2020).

Los esfuerzos de los 30 s y 50 s por impulsar una política de construcción de infraestructura social y productiva no revirtió en gran medida la estructura económica de Tacna.

La crisis económica peruana que alcanzó su cenit en los finales de los años ochenta decantó en una mecánica de relación fundamentada en el pequeño comercio o de menudeo además de la presencia del contrabando fronterizo, impulsado por la creación de la zona franca de Iquique y el deterioro monetario en el Perú. Ya en la última década de la centuria pasada, Perú implementa un mecanismo similar impulsando la Zona Franca Comercial y Turística en Tacna, poniendo a Arica entre ambos espacios de excepción.

A pesar de los cambios y de la redefinición de los tejidos sociales en ambos lados de la frontera, el espacio TacnaArica exhibe aún muchas de las particularidades y potencialidades complementarias que exhibían desde su nacimiento como ciudades y regiones, resultando pertinente evaluar el comportamiento de complementariedad y exclusión que se definen sobre las actividades económicas allí implementadas.

En base a lo anterior el trabajo se plantea:

- Determinar la composición sectorial productiva a nivel intra e interregional, en atención a la incorporación de población económicamente activa, en las regiones Tacna y Moquegua durante el período intercensal 2007-2017.

- Evaluar la especialización y los patrones de producción que exhibieron las regiones Tacna y Moquegua en el período intercensal 2007-2017.

- Analizar el dinamismo económico y estructural observado en las regiones Tacna y Moquegua durante el período intercensal 2007-2017.

\section{MATERIALES Y MÉTODOS}

El tipo de investigación es aplicada, al generar conocimiento para su uso inmediato (en este caso en particular construir información económica agregada para la toma de decisiones de actores decisores).

El enfoque de la investigación es cuantitativa, al estar basada en la información numérica de las variables analizadas. 
El estudio tiene un alcance descriptivo, el cual se encuentra delimitado del modo siguiente:

- Espacio: La jurisdicción del departamento de Tacna y la región Arica-Parinacota

- Tiempo: Para la evaluación de las variables se contempla la disposición de datos correspondientes a los años 2007 y 2017.

En cuanto al diseño de investigación esta es de tipo no experimental seccional descriptivo.

Para determinar la necesidad de información se utilizó la siguiente matriz de operacionalización de variables (tabla 1).

\section{Tabla 1}

Matriz de operacionalización de variables de la investigación

\begin{tabular}{|c|c|c|}
\hline Variable & Dimensión & Indicadores \\
\hline \multirow{3}{*}{$\begin{array}{l}\text { Estructura económica regional } \\
\text { del espacio bifronterizo Tacna- } \\
\text { Arica }\end{array}$} & $\begin{array}{l}\text { Composición sectorial } \\
\text { productiva a nivel intra e } \\
\text { interregional }\end{array}$ & $\begin{array}{ll}\text { - } & \text { Cociente de participación } \\
\text { del sector en la región (Pij) } \\
\text { - Cociente de participación de } \\
\text { la región en el sector (Pji) }\end{array}$ \\
\hline & $\begin{array}{l}\text { Especialización y los patrones } \\
\text { de producción }\end{array}$ & $\begin{array}{ll}\text { - } & \text { Cociente de localización } \\
& \text { (Qij) } \\
\text { - } & \text { Coeficiente } \\
& \text { especialización }\left(\mathrm{Q}_{\mathrm{r}}\right) \\
\text { - } & \begin{array}{l}\text { Cociente de variación del } \\
\text { sector en la región }\left(\mathrm{rV}_{\mathrm{ij}}\right) \\
\text { - }\end{array} \\
\text { Coeficiente } & \mathrm{de} \\
& \text { Reestructuración }\left(\mathrm{CR}_{\mathrm{r}}\right)\end{array}$ \\
\hline & $\begin{array}{l}\text { Dinamismo económico y } \\
\text { estructural }\end{array}$ & $\begin{array}{ll}- & \text { Efecto diferencial }\left(\mathrm{ED}_{\mathrm{j}}\right) \\
- & \text { Efecto estructural }\left(\mathrm{EE}_{\mathrm{j}}\right) \\
- & \text { Efecto total }\end{array}$ \\
\hline
\end{tabular}

\section{Recopilación de datos}

Para formular el análisis se obtuvieron datos básicamente de origen documental, los cuales se encuentran disponibles en acceso abierto a través de las páginas web institucionales de las entidades que lo manejan como: Servicios de Impuestos Internos de Chile (SII), Instituto Nacional de Estadística e Informática del Perú (INEI) y el Instituto Nacional de Estadística de Chile(INE). Entre la información recopilada se tiene:

- Población económicamente activa, según categoría de ocupación del departamento de Tacna (2007), según resultados del Censo Nacional 2007: XI de Población y VI de Vivienda.

- Población económicamente activa, según categoría de ocupación del departamento de Tacna (2017), según resultados del Censo Nacional 2017: XII de Población y VII de Vivienda.

- Población económicamente activa, según categoría de ocupación de la región Arica y Parinacota (2007), según datos del Sistema de Impuestos Internos de Chile.

- Población económicamente activa, según categoría de ocupación de la región Arica y Parinacota (2017), según resultados del Censo Nacional 2017.

\section{Manejo y procesamiento de los datos}

Para la construcción de los resultados, se consideró inicialmente que la información se estructure preferentemente en base a datos censales (con la sola excepción del año 2007 en Chile) y considerando para la clasificación de la Población Económicamente Activa (15 años a más) que laboren en las actividades económicas tipificadas en atención a la Clasificación Industrial Internacional Uniforme versión 4. 
En base a esta información y considerando la propuesta metodológica de Lira y Quiroga (2003), se planteó el siguiente procedimiento de trabajo:

- Inicialmente, se elaboró una ficha de registra de datos de la Población Económicamente Activa ocupada para los años 2007 y 2017, considerando que cada celda es representada por $\mathrm{V}_{\mathrm{ij}}$ que implica el valor de la variable correspondiente al sector "i" y región "j".

- A partir de lo anterior se definió el cociente de participación del sector en la región $\left(\mathrm{P}_{\mathrm{ij}}\right)$, que nos permite conocer la participación porcentual de cada uno los sectores económicos, de modo tal que permite evaluar la especialización absoluta o intraregional. Para su determinación se usó:

$$
P_{i j}=100 \times \frac{V_{i j}}{\sum_{i=1}^{n} V_{i j}}
$$

Los valores de este indicador se encuentran expresados a nivel porcentual.

- Posteriormente, se calculó el cociente de participación de la región en el sector $\left(\mathrm{P}_{\mathrm{j} j \mathrm{j}}\right)$, que representa la participación porcentual de la región en cada uno de los sectores económicos del patrón usado de referencia, de modo que se pueda apreciar la distribución en el conjunto del territorio en análisis y su concentración en relación a dichos sectores.

$$
P_{j i}=100 \times \frac{V_{i j}}{\sum_{j=1}^{m} V_{i j}}
$$

Del mismo modo que el indicador precio, los valores son porcentuales.

- Luego, se determinó el cociente de localización (Qij), que representa la especialización en el conjunto del territorio de análisis en referencia a los sectores económicos presentes en la región. Para su cálculo se hizo uso de la siguiente fórmula:

$$
Q_{i j}=\frac{V_{i j} / \sum_{i=1}^{n} V_{i j}}{\sum_{j=1}^{m} V_{i j} / \sum_{i=1}^{n} \sum_{j=1}^{m} V_{i j}}
$$

La especialización relativa apreciable de cada región en un sector determina un valor proporcional, esto es Qij > 1 .

- Se generó el denominado coeficiente de especialización $\left(\mathrm{Q}_{r}\right)$, el cual mide el grado de similitud de la estructura económica de la región respecto a la estructura económica del patrón de comparación. Para su cuantificación, se utilizó la siguiente expresión:

$$
Q_{r}=\frac{1}{2} \times \sum_{i=1}^{n}\left[\left|\frac{V_{i j}}{\sum_{i=1}^{n} V_{i j}}-\frac{\sum_{j=1}^{m} V_{i j}}{\sum_{i=1}^{n} \sum_{j 01}^{m} V_{i j}}\right|\right]
$$

Este valor se utiliza como medida de la "especialización regional", un indicador cercano a 1 implica una "diversificación regional"; en tanto que un valor cercano a "0" determina una economía especializada en términos relativos. 
- Luego, se calculó el cociente de variación del sector en la región $\left(\mathrm{rV}_{\mathrm{ij}}\right)$, el que representa la variación (para nuestro caso en un período de 10 años) de los sectores económicos presentes en la región. Para tal fin se utilizó:

$$
r V_{i j}=\sqrt[10]{\frac{V_{i j}(2017)}{V_{i j}(2007)}}
$$

Este indicador revela crecimiento $\left(\mathrm{si}_{\mathrm{r}} \mathrm{V}_{\mathrm{ij}}>1\right)$, estancamiento $\left(\mathrm{si} \mathrm{rV}_{\mathrm{ij}}=0\right)$ o caída $\left(\mathrm{si}_{\mathrm{rij}}<1\right)$ para la variable.

- Posteriormente, se calculó el coeficiente de reestructuración $\left(\mathrm{CR}_{\mathrm{r}}\right)$, que determina los posibles cambios en la estructura económica de una región (previendo el uso del mismo período comprendido entre el 2007 y el 2017) en términos de su composición sectorial, dicho valor se obtuvo:

$$
C R_{r}=\frac{1}{2} \times \sum_{i=1}^{n}\left[\left|\frac{V_{i j}(2017)}{\sum_{i=1}^{n} V_{i j}(2017)}-\frac{V_{i j}(2007)}{\sum_{i=1}^{n} V_{i j}(2007)}\right|\right]
$$

Esta variación oscila entre 0 y 1 . Si el coeficiente es igual a 0 , implica que no se han generado cambios significativos en la estructura económica regional. Si el coeficiente fuera 1 , se prevé una reestructuración regional profunda en el período.

- A partir de todo lo anterior, se obtuvo el efecto diferencial $\left(E D_{j}\right)$, que determina si el dinamismo económico de la región contribuyó al desarrollo territorial positivamente, negativamente o si fue neutral. Al respecto, su procedimiento de cálculo plantea:

$$
E D_{j}=\sum_{i=1}^{n}\left[V_{i j}(2017)-V_{i j}(2007) \times \frac{\sum_{j=1}^{m} V_{i j}(2017)}{\sum_{j=1}^{m} V_{i j}(2007)}\right]
$$

El efecto diferencial recoge la dinámica de cada sector “i” en la región “j”” comparada con la dinámica del mismo sector en el patrón de comparación.

- De igual manera se obtuvo el efecto estructural $\left(E_{\mathrm{j}}\right)$, que permite conocer si la composición sectorial de las actividades económicas presentes en la región contribuyó al desarrollo económico del territorio en análisis en forma positiva, negativa o fue neutral en función al patrón de comparación, se utilizó para ello la siguiente fórmula:

$$
E E_{j}=\sum_{i=1}^{n}\left[V_{i j}(2007) \times\left(\frac{\sum_{j=1}^{m} V_{i j}(2017)}{\sum_{j=1}^{m} V_{i j}(2007)}-\frac{\sum_{i=1}^{n} \sum_{j=1}^{m} V_{i j}(2017)}{\sum_{i=1}^{n} \sum_{j=1}^{m} V_{i j}(2007)}\right)\right]
$$

Este efecto permite cuantificar las diferencias acumuladas existentes entre el valor observado y el valor esperado del comportamiento de cada sector económico en los territorios que conforman la región en estudio.

Si se obtienen efectos diferenciales positivos, esto determina que el territorio en análisis presenta mejores condiciones de accesibilidad a los mercados finales o a los recursos productivos, en comparación a otros territorios dedicados a las mismas actividades. 
- Con los dos indicadores anteriores se construye el efecto total, que determina el desarrollo económico de la región evaluando si fue superior, inferior o igual al patrón de comparación. Este se obtiene del modo siguiente:

$$
E T_{j}=\sum_{i=1}^{n} V_{i j}(2017)-\sum_{i=1}^{n} V_{i j}(2007) \times \frac{\sum_{i=1}^{n} \sum_{j=1}^{m} V_{i j}(2017)}{\sum_{i=1}^{n} \sum_{j=1}^{m} V_{i j}(2007)}
$$

El valor "esperado o hipotético" se obtiene aplicando el cociente de variación global (rSR) al valor inicial de la variable (en el año 0 ). El efecto total positivo o negativo refleja un desarrollo económico regional relativo mayor o menor que el patrón de comparación.

Finalmente, y considerando los valores del ETj, EEj y EDj calculados, se puede clasificar el territorio en análisis a partir de su posición relativa en el plano cartesiano tomando en cuenta las siguientes tipologías:

\section{Tabla 2}

Tipologías de territorio según su nivel y ritmo de crecimiento

\begin{tabular}{|c|c|c|c|}
\hline Tipo & EDj & $\mathbf{E E j}$ & Explicación \\
\hline $\begin{array}{c}\text { Tipo I -Ganador } \\
\text { (cuadrante superior } \\
\text { derecho) }\end{array}$ & + & + & $\begin{array}{l}\text { Territorios con mejores condiciones de accesibilidad a los } \\
\text { mercados finales o a los recursos productivos y especializados en } \\
\text { sectores económicos dinámicos. } \\
\text { Se entiende también como territorios dinámicos y con peso } \\
\text { significativo interregional. }\end{array}$ \\
\hline $\begin{array}{c}\text { Tipo II-Convergente } \\
\text { (Cuadrante superior } \\
\text { izquierdo) }\end{array}$ & - & + & $\begin{array}{l}\text { Territorios con limitadas condiciones de accesibilidad a los } \\
\text { mercados finales o a los recursos productivos, pero } \\
\text { especializados en sectores económicos dinámicos. } \\
\text { Se entiende también como territorios poco dinámicos y con peso } \\
\text { significativo interregional. }\end{array}$ \\
\hline $\begin{array}{l}\text { Tipo III-Declinante } \\
\text { (Cuadrante inferior } \\
\text { derecho) }\end{array}$ & + & - & $\begin{array}{l}\text { Territorios con mejores condiciones de accesibilidad a los } \\
\text { mercados finales o a los recursos productivos, pero } \\
\text { especializados en sectores económicos poco dinámicos. } \\
\text { Se entiende también como territorios dinámicos y sin peso } \\
\text { significativo interregional. }\end{array}$ \\
\hline $\begin{array}{l}\text { Tipo IV-Estancado } \\
\text { (Cuadrante inferior } \\
\text { izquierdo) }\end{array}$ & - & - & $\begin{array}{l}\text { Territorios con limitadas condiciones de accesibilidad a los } \\
\text { mercados finales o a los recursos productivos y especializados en } \\
\text { sectores económicos poco dinámicos. } \\
\text { Se entiende también como territorios poco dinámicos y sin peso } \\
\text { significativo interregional. }\end{array}$ \\
\hline
\end{tabular}

Fuente: Lira y Quiroga (2003)

\section{RESULTADOS}

Puede apreciarse que al año 2007, el sector económico que incorporó un mayor volumen de la población económicamente activa, en el conglomerado que aglutina a Tacna y Arica, es la actividad comercial (que en ambas regiones incorpora a 39887 personas, lo que implica $23.2523 \%$ del PEA total), seguidos de la categoría que implica a la agricultura, ganadería, silvicultura y pesca que incorpora a 22754 personas $(13.2645 \%$ del PEA total). Estos dos sectores son también los de mayor absorción de mano de obra en Tacna; en tanto que en Arica y Parinacota, la primera categoría en capacidad de atraer fuerza laboral son los hoteles y restaurantes, seguidos de cerca por la actividad comercial. Puede apreciarse también el mayor peso relativo de la población empleada de Tacna en el espacio que aglutina a las dos regiones. 
Tabla 3

Tacna-Arica: PEA activa según categoría de ocupación (2007 y 2017)

\begin{tabular}{|c|c|c|c|c|c|c|}
\hline \multirow[b]{2}{*}{ Sector / Región } & \multicolumn{3}{|c|}{2007} & \multicolumn{3}{|c|}{2017} \\
\hline & Tacna & Arica & $\begin{array}{c}\text { Total } \\
\text { Sector }\end{array}$ & Tacna & Arica & $\begin{array}{c}\text { Total } \\
\text { Sector }\end{array}$ \\
\hline Agricultura, ganadería, silvicultura y pesca & 19314 & 3440 & 22754 & 21693 & 6293 & 27986 \\
\hline Explotación de minas y canteras & 821 & 112 & 933 & 1731 & 2393 & 4124 \\
\hline Industrias manufactureras & 7526 & 5595 & 13121 & 9556 & 3865 & 13421 \\
\hline Suministro de electricidad, gas y agua & 398 & 1430 & 1828 & 681 & 572 & 1253 \\
\hline Construcción & 9098 & 342 & 9440 & 14736 & 7367 & 22103 \\
\hline Comercio & 32857 & 7030 & 39887 & 39510 & 15086 & 54596 \\
\hline Hoteles y restaurantes & 7885 & 7745 & 15630 & 13019 & 4795 & 17814 \\
\hline Transporte y comunicaciones & 11190 & 2463 & 13653 & 15392 & 9097 & 24489 \\
\hline Intermediación financiera & 789 & 3399 & 4188 & 1850 & 773 & 2623 \\
\hline Actividades inmobiliarias & 5908 & 114 & 6022 & 210 & 200 & 410 \\
\hline Administración pública y defensa & 8310 & 2244 & 10554 & 12952 & 14929 & 27881 \\
\hline Enseñanza & 8417 & 3678 & 12095 & 9231 & 7625 & 16856 \\
\hline Servicios sociales y de salud & 3174 & 2785 & 5959 & 4880 & 4262 & 9142 \\
\hline Otras actividades & 3239 & 2060 & 5299 & 3985 & 4488 & 8473 \\
\hline Hogares privados con servicio doméstico & 3461 & 2712 & 6173 & 2337 & 2089 & 4426 \\
\hline Organizaciones y órganos extraterritoriales & 4 & 17 & 21 & 4 & 6 & 10 \\
\hline Actividad económica no especificada & 3983 & 0 & 3983 & 8664 & 14999 & 23663 \\
\hline Total Región & 126374 & 45166 & 171540 & 160431 & 98839 & 259270 \\
\hline
\end{tabular}

Fuente: Censo Nacional 2007: XI de Población y VI de Vivienda, INEI; Sistema de Impuestos Internos de Chile. Censo Nacional 2017: XII de Población y VII de Vivienda, INEI; Censo Nacional 2017, INE.

Diez años después, las dos categorías más relevantes en la conjunción de ambas regiones no han cambiado, manteniéndose la actividad comercial como la predominante (que implica incorporar a 54596 personas o 21 $0576 \%$ del PEA total), seguida por la agricultura, ganadería, silvicultura y pesca (27 989 personas o $10.7942 \%$ del PEA total); notándose la significativa expansión que ha tenido la categoría administración pública y defensa. En el caso de Arica y Parinacota, es precisamente el comercio la categoría más relevante en la generación de puestos de trabajo, constituyendo con la administración pública y defensa (desplazando a la actividad agropecuaria) las dos categorías que explican casi una tercera parte de su PEA. En el caso de Tacna, las cifras demuestran que el comercio así como la agricultura, ganadería, silvicultura y pesca son las categorías que ocupan los lugares más importantes en absorción de su PEA.

Puede notarse también que al 2007, salvo los sectores suministro de electricidad, gas y agua, intermediación financiera, y organizaciones y órganos extraterritoriales, las demás categorías observan la presencia de una mayor cantidad absoluta de personas ocupadas en la región Tacna. Aspecto que cambia una década después donde la región Arica y Parinacota aprecia un predominio en categorías como explotación de minas y canteras, administración pública y defensa, otras actividades, y organizaciones y órganos extraterritoriales.

$\mathrm{Al}$ analizar la especialización interregional dentro del territorio al 2007, puede apreciarse en el caso de Tacna, que esta es distinguible en categorías como actividades inmobiliarias y construcción. Para el caso de Arica y Parinacota es la intermediación financiera (incluso en un grado mucho mayor al exhibido en Tacna), de igual manera el suministro de electricidad, gas y agua. 
Para el año 2017, Tacna trastoca sus especialidades a favor de sectores como la agricultura, ganadería, silvicultura y pesca, así como los hoteles y restaurantes. Se observa en Arica y Parinacota una enorme contracción en las especialidades que poseía, alterándose las mismas por otras categorías como las organizaciones y órganos extraterritoriales y la explotación de minas y canteras.

\section{Tabla 4}

Determinación del cociente de localización (2007 y 2017)

\begin{tabular}{lcccc}
\hline \multirow{2}{*}{ Sector / Región } & \multicolumn{2}{c}{$\mathbf{2 0 0 7}$} & \multicolumn{2}{c}{$\mathbf{2 0 1 7}$} \\
\cline { 2 - 5 } & Tacna & Arica & Tacna & Arica \\
\hline Agricultura, ganadería, silvicultura y pesca & 1.1522 & 0.5742 & 1.2527 & 0.5898 \\
Explotación de minas y canteras & 1.1945 & 0.4559 & 0.6783 & 1.5221 \\
Industrias manufactureras & 0.7786 & 1.6195 & 1.1507 & 0.7554 \\
Suministro de electricidad, gas y agua & 0.2955 & 2.9711 & 0.8783 & 1.1975 \\
Construcción & 1.3082 & 0.1376 & 1.0774 & 0.8743 \\
Comercio & 1.1182 & 0.6694 & 1.1695 & 0.7248 \\
Hoteles y restaurantes & 0.6848 & 1.882 & 1.1811 & 0.7061 \\
Transporte y comunicaciones & 1.1125 & 0.6852 & 1.0158 & 0.9744 \\
Intermediación financiera & 0.2557 & 3.0825 & 1.1398 & 0.773 \\
Actividades inmobiliarias & 1.3317 & 0.0719 & 0.8278 & 1.2796 \\
Administración pública y defensa & 1.0688 & 0.8075 & 0.7507 & 1.4046 \\
Enseñanza & 0.9446 & 1.1549 & 0.885 & 1.1866 \\
Servicios sociales y de salud & 0.723 & 1.775 & 0.8627 & 1.2229 \\
Otras actividades & 0.8297 & 1.4765 & 0.7601 & 1.3894 \\
Hogares privados con servicio doméstico & 0.761 & 1.6686 & 0.8533 & 1.2381 \\
Organizaciones y órganos extraterritoriales & 0.2586 & 3.0746 & 0.6464 & 1.5739 \\
Actividad económica no especificada & 1.3574 & 0 & 0.5917 & 1.6627 \\
\hline Fuente: Censo Nacionat 2007: XI de Poblacion & $\mathbf{V}$ & & & \\
\hline
\end{tabular}

Fuente: Censo Nacional 2007: XI de Población y VI de Vivienda, INEI; Sistema de Impuestos Internos de Chile. Censo Nacional 2017: XII de Población y VII de Vivienda, INEI; Censo Nacional 2017, INE

En cuanto al coeficiente de especialización, puede observarse que Tacna no ha generado cambios significativos en sus actividades productivas entre el 2007 y 2017. Lo cual se expresa en que dicho indicador (muy cercano a 0) ha cambiado de 0.0988 a 0.0927 en la década analizada. En tanto, Arica y Parinacota ha reducido su estructura asimétrica en relación al patrón del binomio Tacna-Arica, acercándose mucho más a la dinámica común, registrando así de un valor de 0.2765 a 0.1505 entre el 2007 y 2017. 
Palza, E.

Tabla 5

Determinación del coeficiente de especialización (2007 y 2017)

\begin{tabular}{lcccc}
\hline & \multicolumn{2}{c}{$\mathbf{2 0 0 7}$} & \multicolumn{2}{c}{$\mathbf{2 0 1 7}$} \\
\cline { 2 - 5 } \multicolumn{1}{c}{ Sector / Región } & Tacna & Arica & Tacna & Arica \\
\hline Agricultura, ganadería, silvicultura y pesca & 0.0202 & 0.0565 & 0.0273 & 0.0443 \\
Explotación de minas y canteras & 0.0011 & 0.003 & 0.0051 & 0.0083 \\
Industrias manufactureras & 0.0169 & 0.0474 & 0.0078 & 0.0127 \\
Suministro de electricidad, gas y agua & 0.0075 & 0.021 & 0.0006 & 0.001 \\
Construcción & 0.017 & 0.0475 & 0.0066 & 0.0107 \\
Comercio & 0.0275 & 0.0769 & 0.0357 & 0.0579 \\
Hoteles y restaurantes & 0.0287 & 0.0804 & 0.0124 & 0.0202 \\
Transporte y comunicaciones & 0.009 & 0.0251 & 0.0015 & 0.0024 \\
Intermediación financiera & 0.0182 & 0.0508 & 0.0014 & 0.0023 \\
Actividades inmobiliarias & 0.0116 & 0.0326 & 0.0003 & 0.0004 \\
Administración pública y defensa & 0.0042 & 0.0118 & 0.0268 & 0.0435 \\
Enseñanza & 0.0039 & 0.0109 & 0.0075 & 0.0121 \\
Servicios sociales y de salud & 0.0096 & 0.0269 & 0.0048 & 0.0079 \\
Otras actividades & 0.0053 & 0.0147 & 0.0078 & 0.0127 \\
Hogares privados con servicio doméstico & 0.0086 & 0.0241 & 0.0025 & 0.0041 \\
Organizaciones y órganos extraterritoriales & 0.0001 & 0.0003 & 0 & 0 \\
Actividad económica no especificada & 0.0083 & 0.0232 & 0.0373 & 0.0605 \\
\hline$\quad$ Qr & 0.0988 & 0.2765 & 0.0927 & 0.1505 \\
\hline Fuente: Censo Naciona 2007: XI dePoblacion
\end{tabular}

Fuente: Censo Nacional 2007: XI de Población y VI de Vivienda, INEI; Sistema de Impuestos Internos de Chile. Censo Nacional 2017: XII de Población y VII de Vivienda, INEI; Censo Nacional 2017, INE

Las categorías o sectores que determinaron una mayor tasa de crecimiento promedio intercensal en todo el territorio fueron la actividad de explotación de minas y canteras (con un valor de $4.4202 \%$ ), la administración pública y defensa (definiendo una tasa de $2.6417 \%$ ), y la construcción (con una expansión anual del orden de $2.3414 \%$ ). Precisamente estas son las dos primeras actividades que notifican una meteórica expansión en Arica y Parinacota (con tasas de 21.3661 y $21.5409 \%$ ). En el caso de Tacna, la mayor expansión estuvo en categorías como intermediación financiera (con 2.3447 \%) y la explotación de minas y canteras (con 2.1084 \%) 


\section{Tabla 6}

Determinación del coeficiente de variación (2007-2017)

\begin{tabular}{|c|c|c|c|}
\hline Sector / Región & Tacna & Arica & $\mathbf{r V i}$ \\
\hline Agricultura, ganadería, silvicultura y pesca & 1.1232 & 1.8294 & 1.2299 \\
\hline Explotación de minas y canteras & 2.1084 & 21.3661 & 4.4202 \\
\hline Industrias manufactureras & 1.2697 & 0.6908 & 1.0229 \\
\hline Suministro de electricidad, gas y agua & 1.7111 & 0.4000 & 0.6854 \\
\hline Construcción & 1.6197 & 21.5409 & 2.3414 \\
\hline Comercio & 1.2025 & 2.1459 & 1.3688 \\
\hline Hoteles y restaurantes & 1.6511 & 0.6191 & 1.1397 \\
\hline Transporte y comunicaciones & 1.3755 & 3.6935 & 1.7937 \\
\hline Intermediación financiera & 2.3447 & 0.2274 & 0.6263 \\
\hline Actividades inmobiliarias & 0.0355 & 1.7544 & 0.0681 \\
\hline Administración pública y defensa & 1.5586 & 6.6529 & 2.6417 \\
\hline Enseñanza & 1.0967 & 2.0731 & 1.3936 \\
\hline Servicios sociales y de salud & 1.5375 & 1.5303 & 1.5342 \\
\hline Otras actividades & 1.2303 & 2.1786 & 1.5990 \\
\hline Hogares privados con servicio doméstico & 0.6752 & 0.7703 & 0.7170 \\
\hline Organizaciones y órganos extraterritoriales & 1.0000 & 0.3529 & 0.4762 \\
\hline Actividad económica no especificada & 2.1752 & - & 5.9410 \\
\hline $\mathrm{rRj}$ & 1.2695 & 2.1883 & 1.5114 \\
\hline
\end{tabular}

Fuentes: Censo Nacional 2007: XI de Población y VI de Vivienda, INEI; Sistema de Impuestos Internos de Chile. Censo Nacional 2017: XII de Población y VII de Vivienda, INEI; Censo Nacional 2017, INE.

Si se considera que la expansión promedio de crecimiento (entre el 2007 y 2017) de las categorías económicas de Tacna $(1.2695 \%)$ se encuentran por debajo de la tasa de crecimiento poblacional intercensal, esto determina una presión para la reestructura económica, la cual no se aprecia como notoria (definiendo un coeficiente de reestructuración de 0.0995). Por otro lado, en Arica y Parinacota se ha apreciado una mayor expansión en la absorción de fuerza laboral, y se observa también un crecimiento orientado hacia nuevas actividades (lo que se acredita al generar un coeficiente de reestructuración de 0.3793 ). 
Palza, E.

Tabla 7

Determinación del coeficiente de reestructuración (2007-2017)

\begin{tabular}{|c|c|c|}
\hline Sector / Región & Tacna & Arica \\
\hline Agricultura, ganadería, silvicultura y pesca & 0.0176 & 0.0125 \\
\hline Explotación de minas y canteras & 0.0043 & 0.0217 \\
\hline Industrias manufactureras & 0.0000 & 0.0848 \\
\hline Suministro de electricidad, gas y agua & 0.0011 & 0.0259 \\
\hline Construcción & 0.0199 & 0.0670 \\
\hline Comercio & 0.0137 & 0.0030 \\
\hline Hoteles y restaurantes & 0.0188 & 0.1230 \\
\hline Transporte y comunicaciones & 0.0074 & 0.0375 \\
\hline Intermediación financiera & 0.0053 & 0.0674 \\
\hline Actividades inmobiliarias & 0.0454 & 0.0005 \\
\hline Administración pública y defensa & 0.0150 & 0.1014 \\
\hline Enseñanza & 0.0091 & 0.0043 \\
\hline Servicios sociales y de salud & 0.0053 & 0.0185 \\
\hline Otras actividades & 0.0008 & 0.0002 \\
\hline Hogares privados con servicio doméstico & 0.0128 & 0.0389 \\
\hline Organizaciones y órganos extraterritoriales & 0.0000 & 0.0003 \\
\hline Actividad económica no especificada & 0.0225 & 0.1518 \\
\hline $\mathrm{CRr}$ & 0.0995 & 0.3793 \\
\hline
\end{tabular}

Fuente: Censo Nacional 2007: XI de Población y VI de Vivienda, INEI; Sistema de Impuestos Internos de Chile. Censo Nacional 2017: XII de Población y VII de Vivienda, INEI; Censo Nacional 2017, INE

Del análisis del efecto diferencial puede apreciarse que Tacna ganó (entre el 2007 y el 2017) en dinamicidad en sectores como las industrias manufactureras, el suministro de electricidad, gas y agua, intermediación financiera, servicios sociales y de salud, además de organizaciones y órganos extraterritoriales. En tanto que Arica y Parinacota lo hizo en las 13 categorías restantes.

\section{Tabla 8}

Determinación del efecto diferencial (2007-2017)

\begin{tabular}{lcc}
\multicolumn{1}{c}{ Sector / Región } & Tacna & Arica \\
\hline Agricultura, ganadería, silvicultura y pesca & -2.062 & 2.062 \\
Explotación de minas y canteras & -1.898 & 1.898 \\
Industrias manufactureras & 1.858 & -1.858 \\
Suministro de electricidad, gas y agua & 408 & -408 \\
Construcción & -6.566 & 6.566 \\
Comercio & -5.464 & 5.464 \\
Hoteles y restaurantes & 4.032 & -4.032 \\
Transporte y comunicaciones & -4.679 & 4.679 \\
Intermediación financiera & 1.356 & -1.356 \\
Actividades inmobiliarias & -192 & 192 \\
Administración pública y defensa & -9.001 & 9.001 \\
Enseñanza & -2.499 & 2.499 \\
Servicios sociales y de salud & 11 & -11 \\
Otras actividades & -1.194 & 1.194 \\
Hogares privados con servicio doméstico & -145 & 145 \\
Organizaciones y órganos extraterritoriales & 2 & -2 \\
Actividad económica no especificada & -14.999 & 14.999 \\
\hline
\end{tabular}

Fuentes: Censo Nacional 2007: XI de Población y VI de Vivienda, INEI; Sistema de Impuestos Internos de Chile. Censo Nacional 2017: XII de Población y VII de Vivienda, INEI; Censo Nacional 2017, INE. 
En cuanto a la mayor dinámica definida por la dedicación a sectores productivos más competitivos, Tacna aprecia notorias mejoras en reasignaciones a categorías como la administración pública y enseñanza, construcción, transportes y comunicaciones, explotación de minas y canteras, entre otros. Para el caso de Arica y Parinacota, estas se aprecian fundamentalmente en la administración pública y enseñanza.

\section{Tabla 9}

Determinación del efecto estructural (2007-2017)

\begin{tabular}{lcc}
\hline \multicolumn{1}{c}{ Sector / Región } & Tacna & Arica \\
\hline Agricultura, ganadería, silvicultura y pesca & -5.437 & -968 \\
Explotación de minas y canteras & 2.388 & 326 \\
Industrias manufactureras & -3.677 & -2.734 \\
Suministro de electricidad, gas y agua & -329 & -1.181 \\
Construcción & 7.551 & 284 \\
Comercio & -4.687 & -1.003 \\
Hoteles y restaurantes & -2.931 & -2.879 \\
Transporte y comunicaciones & 3.158 & 695 \\
Intermediación financiera & -698 & -3.008 \\
Actividades inmobiliarias & -8.527 & -165 \\
Administración pública y defensa & 9.393 & 2.536 \\
Enseñanza & -991 & -433 \\
Servicios sociales y de salud & 72 & 63 \\
Otras actividades & 284 & 180 \\
Hogares privados con servicio doméstico & -2.750 & -2.155 \\
Organizaciones y órganos extraterritoriales & -4 & -18 \\
Actividad económica no especificada & 17.643 & 0 \\
\hline \multicolumn{1}{c}{ EEj } & 10.458 & -10.458 \\
\hline
\end{tabular}

Fuentes: Censo Nacional 2007: XI de Población y VI de Vivienda, INEI; Sistema de Impuestos Internos de Chile. Censo Nacional 2017: XII de Población y VII de Vivienda, INEI; Censo Nacional 2017, INE.

A partir de todo lo anterior puede establecerse una mejora de región de Arica y Parinacota en la dinámica económica en el espacio territorial "tacnoariqueño" en detrimento de Tacna. En gran medida, ese incremento se explica por una mayor dinamicidad de muchas de sus actividades productivas y a pesar que su composición de la PEA se orienta hacia categorías productivas escasamente competitivas. En el caso de Tacna, y a pesar que se orienta hacia categorías más competitivas, estas no observaron la dinamicidad que impulse en mejor medida su competitividad.

Tabla 10

Determinación del efecto total (2007-2017)

\begin{tabular}{lc}
\hline \multicolumn{1}{c}{ Territorio } & ETj \\
\hline Tacna & -30.574 \\
Arica & 30.574 \\
\hline Fuente: Censo Nacional 2007: XI de Población y VI de Vivienda, INEI; Sistema de \\
Impuestos Internos de Chile. Censo Nacional 2017: XII de Población y VII de Vivienda, \\
INEI; Censo Nacional 2017, INE.
\end{tabular}

Con ello, se puede determinar que la región Tacna puede ser considerada como un territorio de Tipo II, un territorio con limitadas condiciones de accesibilidad a los mercados finales o a los recursos productivos, pero especializados en sectores económicos dinámicos. Esto implica reconocer a Tacna como un territorio poco dinámico, pero con un peso significativo interregional. 
La evaluación también determina a Arica y Parinacota como un territorio con mejores condiciones de accesibilidad a los mercados finales o a los recursos productivos, pero especializados en sectores económicos poco dinámicos. Se entiende también como un territorio dinámico y sin peso significativo interregional.

Tabla 11

Clasificación del territorio, según su nivel y ritmo de crecimiento

\begin{tabular}{|c|c|c|c|c|c|c|}
\hline \multirow[b]{2}{*}{ Territorio } & \multirow{2}{*}{$\begin{array}{c}\text { E. } \\
\text { Diferencial }\end{array}$} & \multirow{2}{*}{$\begin{array}{c}\text { E. } \\
\text { Estructural }\end{array}$} & \multirow{2}{*}{$\begin{array}{c}\text { E. } \\
\text { Total }\end{array}$} & \multicolumn{2}{|c|}{ Comportamiento } & \multirow[b]{2}{*}{ Clasificación } \\
\hline & & & & $\begin{array}{c}\text { E. } \\
\text { Diferencial }\end{array}$ & $\begin{array}{c}\text { E. } \\
\text { Estructural }\end{array}$ & \\
\hline Tacna & -41.032 & 10.458 & -30.574 & Bajo & Alto & Tipo II \\
\hline Arica & 41.032 & -10.458 & 30.574 & Alto & Bajo & Tipo III \\
\hline
\end{tabular}

Fuente: Censo Nacional 2007: XI de Población y VI de Vivienda, INEI; Sistema de Impuestos Internos de

Chile. Censo Nacional 2017: XII de Población y VII de Vivienda, INEI; Censo Nacional 2017, INE

\section{DISCUSIÓN}

Como plantean Lira y Quiroga (2003), el desarrollo de cada "región" o "localidad" depende fundamentalmente de las tendencias de crecimiento y desarrollo, que se dan en las otras regiones o localidades, ya que estas son subsistemas muy abiertos. Para el binomio Tacna-Arica, esto resulta evidente considerando además el historial de vinculación y complementariedad que ha exhibido durante varios siglos.

La lejanía de los centros hegemónicos (capitales nacionales de cada país) determina evidentemente la sensación entre sus pobladores de una condición de abandono, y trazan a partir de ello un reclamo y planteamiento constante de implementación de políticas de promoción del desarrollo, contemplando como principal argumento de pedido la condición de ubicación en una frontera particular y especial para cada país.

Esto ha planteado la implementación de mecanismos que para Gonzáles (2015) propone una relación hegemonismo-revanchismo; en ella cada población se ve a sí misma como un conglomerado virtuoso y pacífico en contraparte con una mirada de agresivo y hostil para con el vecino. Las políticas implementadas proponen precisamente un acto reivindicatorio o de mantención de la condición de subordinante.

Sin embargo, las actividades productivas predominantes en la región fronteriza chilena siguen siendo la minería (extracción de cobre, cloruro de sodio y azufre) así como la pesca, turismo y comercio, las cuales han incorporado mejoras a partir del aumento en sus precios entre el 2007 y el 2010. La implementación de beneficios tributarios para zonas extremas (Ley 20.635) planteaba beneficios a las inversiones que se asentasen en la región Arica y Parinacota; así como el mayor desembolso de inversión pública ha impulsado, como se aprecia en los resultados, una mayor incorporación de mano de obra en la labor productiva.

Tacna posee también una economía estructurada sobre la actividad productiva, fundamentalmente de tipo extractiva (impulsada por la agricultura y minería), pero también de la actividad comercial y turística, la cual viene incorporando una mayor asignación de su mano de obra; sin embargo, dado que estas no han tenido un impulso significativo, esto explicaría la no mejora en la dinámica territorial.

Las suspicacias aún persistentes entre ambos países impiden la implementación de políticas fronterizas multiescalares que promuevan una mayor interacción entre las instancias de gobiernos subnacionales con sus gobiernos nacionales, propiciando una mayor articulación en la toma de decisiones que impacten con beneficios simultáneos a los territorios de ambos lados de la frontera (Koff, 2008). La habitual complementariedad de ambas regiones puede, adecuadamente canalizada, determinar acciones con mutuos beneficios.

\section{CONCLUSIONES}

En base al período analizado, se aprecia que la economía de Arica incorpora la mayor parte de su mano de obra en actividades como la minería (extracción de cobre, cloruro de sodio y azufre), pesca, turismo y comercio; mostrando adicionalmente un crecimiento explicado fundamentalmente por la mejora de los precios internacionales de los bienes generados en estos sectores. En tanto, en Tacna se aprecia una economía que 
fundamenta la incorporación laboral por los sectores extractivos (agricultura y minería), pero también explicada por la actividad comercial y turística, sectores que además vienen observando un mayor crecimiento.

La denominada región Arica y Parinacota determinó un importante crecimiento en sectores como el minero y el sector de la administración pública. En este último caso, explicado por la implementación de una serie de medidas gubernamentales orientadas a brindar beneficios en las zonas fronterizas de Chile. En el caso de Tacna, la mayor expansión estuvo en categorías como intermediación financiera así como la explotación de minas y canteras.

Tacna ganó en el período de análisis una mayor dinamicidad en sectores como las industrias manufactureras; el suministro de electricidad, gas y agua, e intermediación financiera; en tanto que Arica tuvo un actuar más dinámico en la administración pública, construcción y comercio. En cuanto a la reasignación de mano de obra a sectores más competitivos, Tacna aprecia reorientaciones a actividades como la administración pública y enseñanza, construcción, transportes y comunicaciones, explotación de minas y canteras, entre otros. En tanto que, en el caso de Arica y Parinacota, estas se aprecian fundamentalmente hacia la administración pública y enseñanza. En base a lo anterior se puede apreciar a Tacna como un territorio poco dinámico, pero con una alta presencia de su PEA en sectores más dinámicos. Arica en contraste se exhibe como un territorio con mejores condiciones de accesibilidad a los mercados finales o a los recursos productivos, pero especializados en sectores económicos poco dinámicos.

\section{REFERENCIAS}

Banco Central de Reserva del Perú. (s.f.). Caracterización del departamento de Tacna. https://www.bcrp.gob.pe/docs/Sucursales/Arequipa/Tacna-Caracterizacion.pdf

Biblioteca del Congreso Nacional de Chile. (s.f.). Biblioteca del Congreso Nacional de Chile. Obtenido de Información Territorial: https://www.bcn.cl/siit/nuestropais/nuestropais/region15/

Contreras, Y., Tapia, M., \& Liberona, N. (2017). Movilidades y prácticas sociales fronterizas entre Tacna y Arica. Del sentido de la frontera a la transfronterizidad entre ciudades. Diálogo andino, 127-141.

Díaz, A., \& Bustos, R. (2020). Crisis en la frontera: Percepción de abandono en Tacna y Arica luego del tratado de 1929. Diálogo andino, 153-160.

Dilla, H., \& Álvarez, C. (2018). Arica/Tacna: Los circuitos económicos de un complejo urbano transfronterizo. Diálogo andino, 99-109.

Gárate, J. (2016). Flujos de visitantes y turistas 2011-2015. I Congreso de Desarrollo Fronterizo AricaTacna: ¿la frontera como oportunidad para el desarrollo? Arica: Universidad Arturo Prat.

Gonzáles, A. (2015). Psicología política de la integración fronteriza. Liberabit, 269-287.

Instituto Nacional de Estadística e Informática. (1998). INEI. Compendio estadístico 1996-1997. http://proyectos.inei.gob.pe/web/biblioineipub/bancopub/Est/LIB0170/n00.htm

Koff, H. (2008). La política fronteriza comparada y las estructuras del poder. Estudios Políticos, 119134.

Lira, L., \& Quiroga, B. (2003). Técnicas de análisis regional. Instituto Latinoamericano y del Caribe de Planificación Económica y Social (ILPES).

Moreno, A. (1977). El corregidor de indios y la economía peruana del siglo XVIII (los repartos forzosos de mercaderías. Madrid: Consejo Superior de Investigaciones Científicas, Instituto Gonzalo Fernández de Oviedo.

Pizarro, E., \& Ríos, W. (2005). Entre franquicias y beneficios: Una apuesta del gobierno para el desarrollo regional de Arica (1953). Diálogo andino, 101-111.

Registro Nacional de Identificación y Estado Civil. (2021). Portal estadístico RENIEC. Obtenido de Población identificada: https://portales.reniec.gob.pe/web/estadistica/identificada

Rosenblitt, J. (2013). Centralidad geográfica, marginalidad política: La región de Tacna-Arica y su comercio 1778-1841. Ediciones de la Dirección de Bibliotecas, Archivos y Museos.

Rosenblitt, J. (2018). Los circuitos comerciales en el eje Tacna-Arica durante la temprana República peruana, 1840-1863. Una aproximación a los comerciantes intermedios. Revista del Instituto Riva Agüero, 203-242. 
Superintendencia Nacional de Administración Tributaria. (29 de Setiembre de 2020). SUNAT. Obtenido de Nota tributaria y aduanera: http://www.sunat.gob.pe/estadisticasestudios/ingresosrecaudados.html

Superintendencia Nacional de Migraciones. (2020). Boletín estadístico migratorio. Obtenido de Movimiento migratorio (2016-2020): https://datastudio.google.com/u/0/reporting/b47403a5b5fb-4a7a-8ed1-e0dd3e57274f/page/sh9aB

Tapia, M. (2015). Frontera, movilidad y circulación reciente de peruanos y bolivianos en el norte de Chile. Estudios Atacameños Arqueología y Antropología Surandina, 195-213.

Tapia, M., Liberona, N., \& Contreras, Y. (2017). El surgimiento de un territorio circulatorio en la frontera chileno-peruana: estudio de las prácticas socio-espaciales fronterizas. Revista de Geografia Norte Grande, 117-141.

Yábar, B. (2019). Una aproximación a la actividad comercial de Tacna y Arica hacia 1840 durante el gobierno de Agustín Gamarra, un enfoque oficial - regional. Iberoamerican Business Journal, 69-84. 